\title{
Synthesis of the C29-C37 Bicyclic Ether Core of (+)-Sorangicin A
}

\author{
Michael T. Crimmins* and Matthew W. Haley
}

\author{
Department of Chemistry, Venable and Kenan Laboratories of Chemistry, University of North Carolina at \\ Chapel Hill, Chapel Hill, North Carolina 27599-3290 \\ Crimmins@email.unc.edu
}

Supporting Information: Experimental Procedures 


\section{Experimental Procedures}

Infrared (IR) spectra were obtained using a JACSO FT/IR 460-plus spectrometer. Proton and carbon nuclear magnetic resonance $\left({ }^{1} \mathrm{H}\right.$ and ${ }^{13} \mathrm{C}$ NMR $)$ spectra were recorded on the following instruments: Bruker model DRX $400\left({ }^{1} \mathrm{H}\right.$ at $400 \mathrm{MHz} ;{ }^{13} \mathrm{C}$ at $\left.100 \mathrm{MHz}\right)$ and Bruker model AMX $300\left({ }^{1} \mathrm{H}\right.$ at $\left.300 \mathrm{MHz}\right)$. Chemical shifts are reported in ppm with residual undeuterated solvent peaks as internal reference for ${ }^{1} \mathrm{H}$ NMR: $\mathrm{CHCl}_{3}(7.26 \mathrm{ppm})$ and deuterated solvent shifts for ${ }^{13} \mathrm{C}$ NMR: $\mathrm{CDCl}_{3}$ (77.0). Multiplicities are reported as (s) singlet, (d) doublet, (t) triplet, (q) quartet and $(\mathrm{m})$ multiplet. Unresolved, overlapping resonances are reported as "band". Coupling constants $(J)$ are given in Hz. Optical rotations were determined using a JACSO P1010 polarimeter. Mass spectra were obtained using a Micromass Quattro II (triple quad) instrument with nanoelectrospray ionization. Thin layer chromatography (TLC) was conducted on silica gel $60 \mathrm{~F}_{254}$ TLC plates purchased from VWR. Flash chromatography was carried out using silica gel $\left(60 \AA, 40\right.$ to 63 m) purchased from SiliCycle. Diethyl ether $\left(\mathrm{Et}_{2} \mathrm{O}\right)$, tetrahydrofuran (THF), and dichloromethane $\left(\mathrm{CH}_{2} \mathrm{Cl}_{2}\right)$ were dried by passing through a column of neutral alumina under nitrogen immediately prior to use. Alkylamines and benzene were distilled from calcium hydride immediately prior to use. Magnesium bromide diethyl etherate was used as received from Aldrich Chemical Co. and stored in a glove box. Titanium (IV) isopropoxide was distilled under reduced pressure and stored in a desiccator. All other reagents and solvents were used as received from the manufacturer. All air and water sensitive reactions were performed under a positive flow of argon in flame dried flasks.
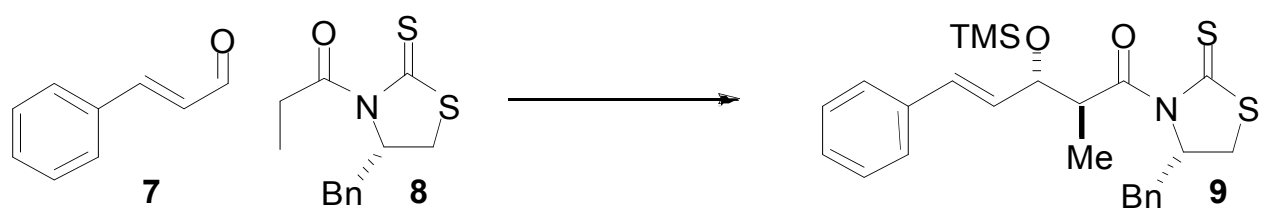

To $\mathrm{MgBr}_{2} \cdot \mathrm{Et}_{2} \mathrm{O}$ (1.46 g, $\left.5.65 \mathrm{mmol}\right)$ was added EtOAc (100 mL, HPLC grade), propionylthiazolidinethione $8(10.0 \mathrm{~g}, 37.7 \mathrm{mmol})$, distilled cinnamaldehyde $(5.22 \mathrm{~mL}, 41.4$ mmol), distilled triethylamine $(10.5 \mathrm{~mL}, 75.4 \mathrm{mmol})$, and distilled TMSCl $(7.22 \mathrm{~mL}, 56.5$ mmol). The milky yellow reaction mixture was stirred for $40 \mathrm{~h}$. The reaction mixture was then filtered through a plug of silica gel and flushed with $\mathrm{Et}_{2} \mathrm{O}$. The eluent was evaporated and purified via flash chromatography ( $2 \%$ EtOAc/hexanes/trace triethylamine) to yield $14.6 \mathrm{~g}$ of aldol-adduct 9 (83\%). ${ }^{1} \mathrm{H}$ NMR (400 MHz, $\left.\mathrm{CDCl}_{3}\right) \delta$ 7.38-7.20 (band, $\left.10 \mathrm{H}\right), 6.55$ (d, $J=16.0$, $1 \mathrm{H}), 6.08(\mathrm{dd}, J=16.0,8.0,1 \mathrm{H}), 5.10(\mathrm{ddd}, J=10.6,6.7,3.8,1 \mathrm{H}), 4.47(\mathrm{dd}, J=8.6,1 \mathrm{H}), 4.34$ (dddd, $J=9.1,6.7,6.7,6.7,1 \mathrm{H}), 3.36-3.27$ (band, $2 \mathrm{H}$ ), 3.09 (dd, $J=13.2,10.8,1 \mathrm{H}), 2.92$ (d, $J=$ $11.4,1 \mathrm{H}), 1.18(\mathrm{~d}, J=6.7,3 \mathrm{H}), 0.08(\mathrm{~s}, 9 \mathrm{H}) ;{ }^{13} \mathrm{C}$ NMR $\left(100 \mathrm{MHz}, \mathrm{CDCl}_{3}\right) \delta 201.10,177.93$, 136.77, 136.38, 132.57, 130.10, 129.46, 128.92, 128.64, 127.91, 127.15, 126.55, 78.48, 69.41, 46.37, 36.55, 32.95, 14.09, 0.45; IR (thin film) v 3027(m), 2957(m), 1950(w), 1880(w), 1811(w), 1697(s), 1494(m), 1453(m), 1363(s), 1342(s), 1292(m), 1262(s), 1226(m), 1190(s), 1166(s), 1137(m), 1111(m), 1053(s); $[\alpha]^{22}{ }_{\mathrm{D}}=+310\left(c=1.4, \mathrm{CH}_{2} \mathrm{Cl}_{2}\right) .{ }^{1}$ 


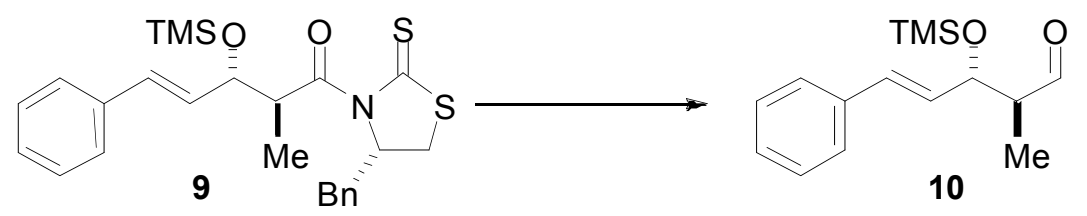

A solution of thione $9(500 \mathrm{mg}, 1.06 \mathrm{mmol})$ in $\mathrm{CH}_{2} \mathrm{Cl}_{2}(8.5 \mathrm{~mL})$ was cooled to $-78^{\circ} \mathrm{C}$. A solution of $i-\mathrm{Bu}_{2} \mathrm{AlH}$ in hexanes $(\sim 1 \mathrm{M})$ was added dropwise until the yellow color disappeared $(1.2 \mathrm{~mL}, \sim 1.2 \mathrm{mmol})$. A saturated solution of sodium potassium tartrate $(4 \mathrm{~mL})$ was added and the mixture was stirred and warmed to rt. Water $(10 \mathrm{~mL})$ and $\mathrm{CH}_{2} \mathrm{Cl}_{2}(10 \mathrm{~mL})$ were added and the heterogeneous mixture was stirred vigorously for $1 \mathrm{~h}$. The layers were separated and the aqueous layer was extracted with $\mathrm{CH}_{2} \mathrm{Cl}_{2}(2 \mathrm{x})$. The organic solution was dried $\left(\mathrm{Na}_{2} \mathrm{SO}_{4}\right)$ and evaporated. The crude oil was brought up in hexanes, whereupon (S)-4-(phenylmethyl)-2thiazolidinethione precipitated as a white solid. The solid was filtered off and washed with additional hexanes. The combined hexanes solution was evaporated and purified via flash chromatography (5\% EtOAc/hexanes/trace triethylamine) to yield $200 \mathrm{mg}$ of aldehyde $\mathbf{1 0}(76 \%)$. ${ }^{1} \mathrm{H}$ NMR $\left(300 \mathrm{MHz}, \mathrm{CDCl}_{3}\right) \delta 9.81(\mathrm{~d}, J=2.3,1 \mathrm{H}), 7.42-7.22$ (band, 5H), $6.58(\mathrm{~d}, J=15.9,1 \mathrm{H})$, $6.20(\mathrm{dd}, J=15.9,7.0,1 \mathrm{H}), 4.48(\mathrm{dd}, J=6.9,6.9,1 \mathrm{H}), 2.60(\mathrm{ddddd}, J=4 \times 7.0,2.3,1 \mathrm{H}), 1.08(\mathrm{~d}$, $J=7.0,3 \mathrm{H}), 0.15(\mathrm{~s}, 9 \mathrm{H}) ;{ }^{13} \mathrm{C} \mathrm{NMR}\left(100 \mathrm{MHz}, \mathrm{CDCl}_{3}\right) \delta 204.30,136.31,131.46,129.93$, 128.58, 127.80, 126.47, 75.02, 52.45, 10.67, 0.24; IR (thin film) v 3028(w), 2959(m), 2876(w), 2727(w), 1948(w), 1877(w), 1803(w), 1729(s), 1496(w), 1450(w), 1252(s), 1124(m), 1061(s); $[\alpha]_{\mathrm{D}}^{22}=+87\left(c=2.9, \mathrm{CH}_{2} \mathrm{Cl}_{2}\right)$; MS (ESI) calculated for $\mathrm{C}_{15} \mathrm{H}_{22} \mathrm{O}_{2} \mathrm{Si}[\mathrm{M}+\mathrm{Na}]^{+}: 285.1$, found 285.1 .

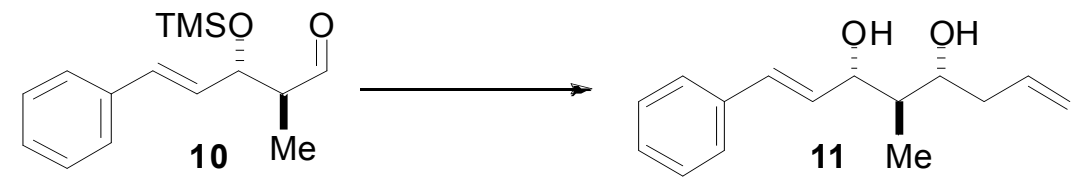

(-)- $\alpha$-Pinene $(1.36 \mathrm{~mL}, 8.54 \mathrm{mmol})$ was added to THF $(1.1 \mathrm{~mL})$. The reaction flask was placed in a rt water bath and $\mathrm{BH}_{3} \cdot \mathrm{SMe}_{2}(0.34 \mathrm{~mL}, 3.56 \mathrm{mmol})$ was added while rapidly stirring the solution. Stirring was ceased immediately following addition and the reaction mixture was allowed to stand for $18 \mathrm{~h}$. The reaction mixture was cooled to $0{ }^{\circ} \mathrm{C}$, after $1 \mathrm{~h}$ the supernatant liquid was then transferred via cannula out of the flask and the remaining white crystals were washed with distilled pentanes $(2 \times 5 \mathrm{~mL})$. The crystals were dried under high vacuum for $0.5 \mathrm{~h}$ $(0.950 \mathrm{~g}, 3.32 \mathrm{mmol})$, and brought up in $1.8 \mathrm{~mL}$ ether and the solution cooled to $4{ }^{\circ} \mathrm{C}$. Methanol $(0.135 \mathrm{~mL}, 3.32 \mathrm{mmol})$ was added slowly as to not increase the temperature above $7{ }^{\circ} \mathrm{C}$. The reaction mixture was stirred at $0{ }^{\circ} \mathrm{C}$ until no white solids remained $(\sim 2 \mathrm{~h})$. Allyl magnesium bromide $\left(3.32 \mathrm{~mL}\right.$ of $1 \mathrm{M}$ solution in $\left.\mathrm{Et}_{2} \mathrm{O}\right)$ was added dropwise. Following addition, the reaction was stirred for $1 \mathrm{~h}$ at $\mathrm{rt}$ then cooled to $-78^{\circ} \mathrm{C}$. Aldehyde $10(200 \mathrm{mg}, 0.76 \mathrm{mmol})$ in ether $(2 \mathrm{~mL})$ was cooled to $-78{ }^{\circ} \mathrm{C}$ and added dropwise to the reaction over 15 min with a jacketed addition funnel. The reaction was complete after 15 min according to NMR analysis. A mixture of $3 \mathrm{~N}$ $\mathrm{NaOH}$ and $30 \% \mathrm{H}_{2} \mathrm{O}_{2}(1 \mathrm{~mL}, 1: 2)$ was added and the reaction mixture was heated to reflux for 4 h. Water $(10 \mathrm{~mL})$ and ether $(10 \mathrm{~mL})$ were added to the solution and the phases were separated. The aqueous layer was extracted with ether $(3 \mathrm{x} 10 \mathrm{~mL})$. The organics were combined and the solvent was evaporated. To a solution of the crude oil in THF $(5 \mathrm{~mL})$ was added $10 \% \mathrm{HCl}(1$ $\mathrm{mL})$ and stirred for $1 \mathrm{~h}$. Water $(10 \mathrm{~mL})$ and ether $(10 \mathrm{~mL})$ were added to the solution and the 
phases were separated. The aqueous layer was extracted with ether $(3 \times 10 \mathrm{~mL})$. The organics were combined, dried $\left(\mathrm{MgSO}_{4}\right)$, and the solvent evaporated. The crude oil was purified via flash chromatography (40\% EtOAc/hexanes) to yield $159 \mathrm{mg}$ of diol $11(90 \%)$ as a colorless oil. ${ }^{1} \mathrm{H}$ NMR (400 MHz, $\left.\mathrm{CDCl}_{3}\right) \delta$ 7.40-7.23 (m, 5H), $6.58(\mathrm{~d}, J=15.9,1 \mathrm{H}), 6.21(\mathrm{dd}, J=15.9,7.7$, $1 \mathrm{H}), 5.89$ (m, 1H), 5.20-5.16 (band, $2 \mathrm{H}), 4.29$ (dd, $J=7.8,7.8,1 \mathrm{H}), 3.69$ (ddd, $J=8.3,8.3,3.1$, 1H), 3.46 (bs, $1 \mathrm{H}), 3.15$ (bs, $1 \mathrm{H}), 2.54-2.42(\mathrm{dm}, J=14.1,2 \mathrm{H}), 2.19$ (ddd, $J=15.5,8.2,8.2$, $1 \mathrm{H}), 1.77(\mathrm{dq}, J=15.1,7.0,1 \mathrm{H}), 0.84(\mathrm{~d}, J=6.9,3 \mathrm{H}) ;{ }^{13} \mathrm{C}$ NMR $\left(100 \mathrm{MHz}, \mathrm{CDCl}_{3}\right) \delta 136.44$, 134.24, 131.80, 130.52, 128.39, 127.56, 126.35, 118.46, 77.84, 74.81, 43.28, 39.40, 12.85; IR $($ thin film) $v$ 3334(br), 3079(m), 3027(m), 2975(s), 2899(s), 1950(w), 1875(w), 1816(w), 1640(w), 1494(m), 1449(m), 1435(m), 1378(w), 1330(m), 1109(m); $[\alpha]^{23}=-31(c=2.0$, $\mathrm{CH}_{2} \mathrm{Cl}_{2}$ ); MS (ESI) calculated for $\mathrm{C}_{15} \mathrm{H}_{20} \mathrm{O}_{2}[\mathrm{M}+\mathrm{H}]^{+}:$233.2, found 232.9.

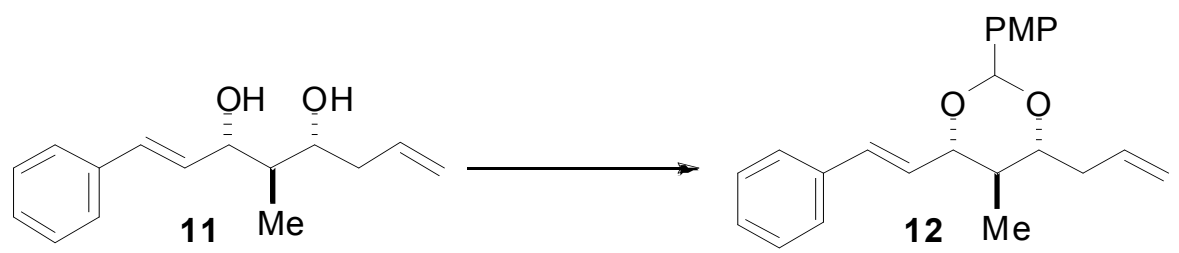

To a solution of diol $11(236 \mathrm{mg}, 1.02 \mathrm{mmol})$ in dry benzene $(10 \mathrm{~mL})$ was added $p$ methoxyphenyl dimethyl acetal $(0.34 \mathrm{~mL}, 2.04 \mathrm{mmol})$ and a spatula tip of pyridinium $p$ toluenesulfonate. The homogeneous solution was stirred for $1.5 \mathrm{~h}$. Ether $(20 \mathrm{~mL})$ and saturated $\mathrm{NaHCO}_{3}(20 \mathrm{~mL})$ were added and the mixture was extracted with ether $(2 \mathrm{x})$. The combined organics was dried $\left(\mathrm{Na}_{2} \mathrm{SO}_{4}\right)$, evaporated, and purified via flash chromatography (5\% EtOAc/hexanes) to yield $337 \mathrm{mg}$ of white crystalline acetal $12(94 \%) .{ }^{1} \mathrm{H}$ NMR (400 MHz, $\left.\mathrm{CDCl}_{3}\right) \delta$ 7.42-7.21 (band, 5H), $7.19\left(\mathrm{AB}, v_{\mathrm{AB}}=233, J=8.6,4 \mathrm{H}\right), 6.80(\mathrm{~d}, J=15.9,1 \mathrm{H}), 6.24$ (dd, $J=15.9,7.6,1 \mathrm{H}), 6.03$ (dddd, $J=17.1,10.2,6.9,6.9,1 \mathrm{H}), 5.62$ (s, 1H), 5.16-5.10 (band, 2H), 4.07 (dd, $J=9.4,9.4,1 \mathrm{H}), 3.80$ (s, 3H), 3.61 (ddd, $J=9.9,7.1,3.1,1 \mathrm{H}), 2.62-2.52$ (band, $2 \mathrm{H}), 2.38(\mathrm{ddd}, J=14.5,7.1,7.1,1 \mathrm{H}), 1.72(\mathrm{ddq}, J=10.0,10.0,6.7,1 \mathrm{H}), 0.88(\mathrm{~d}, J=6.7,3 \mathrm{H})$; ${ }^{13} \mathrm{C}$ NMR $\left(100 \mathrm{MHz}, \mathrm{CDCl}_{3}\right) \delta$ 159.82, 136.50, 134.55, 133.36, 131.23, 128.49, 127.78, 127.66, $127.48,126.57,116.85,113.53,100.26,83.61,81.62,55.26,37.96,37.24,12.21$; IR (thin film) $v$ 2975(m), 2933(m), 2906(m), 2837(s), 2032(w), 1953(w), 1889(w), 1803(w), 1615(s), 1518(s), 1495(m), 1452(m), 1430(m), 1397(s), 1367(m), 1337(s), 1302(s), 1250(s), 1172(s), 1135(s), 1073(s), 1035(s), 1002(s); $[\alpha]^{25}{ }_{\mathrm{D}}=+5.9\left(c=1.6, \mathrm{CH}_{2} \mathrm{Cl}_{2}\right)$; MS (ESI) calculated for $\mathrm{C}_{23} \mathrm{H}_{26} \mathrm{O}_{3}$ $[\mathrm{M}+\mathrm{H}]^{+}: 350.18$, found 350.3 .

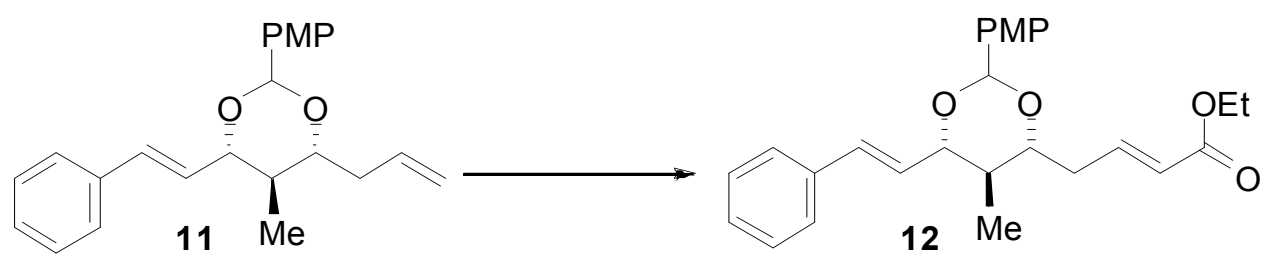

To a solution of terminal olefin $11(0.046 \mathrm{mg}, 0.13 \mathrm{mmol})$ in degassed $\mathrm{CH}_{2} \mathrm{Cl}_{2}(5 \mathrm{~mL})$ was added distilled ethyl acrylate $(0.315 \mathrm{~mL}, 2.89 \mathrm{mmol})$ and the Grubbs second generation catalyst $\left[\mathrm{Cl}_{2}\left(\mathrm{Cy}_{3} \mathrm{P}\right)(\mathrm{IMes}) \mathrm{Ru}=\mathrm{CHPh}\right](6.0 \mathrm{mg}, 0.007 \mathrm{mmol})$. The solution was allowed to stir for 
$14 \mathrm{~h}$ at room temperature and then exposed to air for several hours. The solvent was evaporated the crude black oil was purified via column chromatography (7.5\% EtOAc/hexanes) to yield 53 mg of white crystalline enonoate $12(95 \%) .{ }^{1} \mathrm{H} \mathrm{NMR}\left(400 \mathrm{MHz}, \mathrm{CDCl}_{3}\right) \delta$ 7.42-7.22 (band, $\left.5 \mathrm{H}\right)$, $7.19\left(\mathrm{AB}, v_{\mathrm{AB}}=228.1, J=8.7,4 \mathrm{H}\right), 7.13(\mathrm{ddd}, J=15.7,7.3,7.3,1 \mathrm{H}), 6.69(\mathrm{~d}, J=15.9,1 \mathrm{H})$, $6.23(\mathrm{dd}, J=15.9,7.6,1 \mathrm{H}), 5.63(\mathrm{~s}, 1 \mathrm{H}), 4.21(\mathrm{q}, J=7.1,2 \mathrm{H}), 4.08(\mathrm{dd}, J=9.6,7.9,1 \mathrm{H}), 4.05$ (s, 3H), 3.69 (ddd, $J=10.1,7.2,3.0,1 \mathrm{H}), 2.73-2.64$ (band, $1 \mathrm{H}$ ), 2.53 (ddd, $J=15.0,7.2,7.2$, $1 \mathrm{H}), 1.70(\mathrm{ddq}, J=10.0,10.0,6.6,1 \mathrm{H}), 1.31(\mathrm{t}, J=7.1,3 \mathrm{H}), 0.89(\mathrm{~d}, J=6.8,3 \mathrm{H}) ;{ }^{13} \mathrm{C}$ NMR $\left(100 \mathrm{MHz} \mathrm{CDCl}_{3}\right) \delta 166.21,159.75,144.63,136.24,133.45,130.74,128.38,127.73,127.36$, $127.17,126.45,123.45,113.41,100.17,83.40,80.59,60.09,55.13,37.98,35.50,14.12,12.11$; IR (thin film) $v 2977(\mathrm{~m}), 2934(\mathrm{~m}), 2903(\mathrm{~m}), 2838(\mathrm{~m}), 2032(\mathrm{w}), 1954(\mathrm{w}), 1893(\mathrm{w}), 1715(\mathrm{~s})$, $1655(\mathrm{~m}), 1615(\mathrm{~m}), 1518(\mathrm{~s}), 1453(\mathrm{~m}), 1398(\mathrm{~m}), 1367(\mathrm{~m}), 1303(\mathrm{~s}), 1251(\mathrm{~s}), 1173(\mathrm{~s}), 1138(\mathrm{~s})$, 1073(s), 1035(s); $[\alpha]^{24}=+30\left(c=1.1, \mathrm{CH}_{2} \mathrm{Cl}_{2}\right)$; MS (ESI) calculated for $\mathrm{C}_{26} \mathrm{H}_{30} \mathrm{O}_{5}[\mathrm{M}+\mathrm{H}]^{+}$: 422.2, found 422.2 .

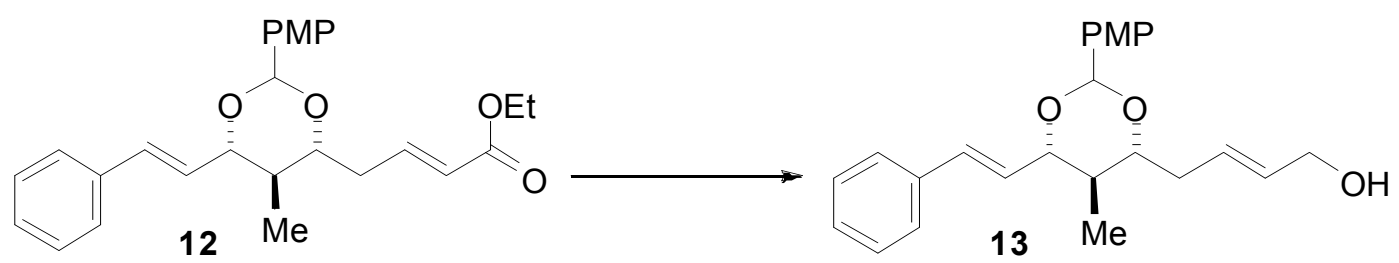

A solution of enoate $12(550 \mathrm{mg}, 1.30 \mathrm{mmol})$ in $\mathrm{CH}_{2} \mathrm{Cl}_{2}(22 \mathrm{~mL})$ was cooled to $-78{ }^{\circ} \mathrm{C}$. A solution of $i-\mathrm{Bu}_{2} \mathrm{AlH}$ in hexanes $(3.90 \mathrm{~mL}, \sim 1 \mathrm{M})$ was added and the reaction mixture was stirred for $15 \mathrm{~min}$. The reaction mixture was quenched with acetone $(10 \mathrm{~mL})$. A saturated solution of sodium potassium tartrate $(10 \mathrm{~mL})$ was added. The heterogeneous mixture was warmed to room temperature and stirred vigorously for $4 \mathrm{~h}$. The mixture was extracted with ether $(3 \mathrm{x})$. The combined organics was dried $\left(\mathrm{Na}_{2} \mathrm{SO}_{4}\right)$ and concentrated in vacuo. The crude product was purified via flash chromatography (40\% EtOAc/hexanes) to yield $462 \mathrm{mg}$ of white crystalline allylic alcohol $13(93 \%) .{ }^{1} \mathrm{H}$ NMR (400 MHz, $\left.\mathrm{CDCl}_{3}\right) \delta$ 7.42-7.20 (band, 5H), 7.19 $\left(\mathrm{AB}, v_{\mathrm{AB}}=229, J=8.7,4 \mathrm{H}\right), 6.67(\mathrm{~d}, J=15.9,1 \mathrm{H}), 6.22(\mathrm{dd}, J=15.9,7.6,1 \mathrm{H}), 5.90(\mathrm{ddd}, J=$ $15.5,6.9,6.9,1 \mathrm{H}), 5.75(\mathrm{ddd}, J=15.5,5.8,5.8), 5.61(\mathrm{~s}, 3 \mathrm{H}), 4.13(\mathrm{~d}, J=5.3,2 \mathrm{H}), 4.06(\mathrm{dd}, J=$ 9.7, 7.8, 1H), $3.80(\mathrm{~s}, 3 \mathrm{H}), 3.59(\mathrm{ddd}, J=9.9,7.0,2.9,1 \mathrm{H}), 2.60-2.50$ (band, $1 \mathrm{H}), 2.37$ (ddd, $J=$ 14.4, 7.3, 7.3, 1H), 1.69 (ddq, $J=10.0,10.0,6.7,1 \mathrm{H}), 1.28(\mathrm{bs}, 1 \mathrm{H}), 0.87(\mathrm{~d}, J=6.7,3 \mathrm{H}) ;{ }^{13} \mathrm{C}$ NMR $\left(100 \mathrm{MHz}, \mathrm{CDCl}_{3}\right) \delta 159.69,136.31,133.26,131.27,130.99,128.41,128.34,127.65$, $127.40,127.33,126.42,113.39,100.14,83.44,81.46,63.54,55.11,37.78,35.43,12.09$; IR (thin film) v 3408(br), 2962(m), 2929(s), 2841(s), 2032(w), 1954(w), 1889(w), 1614(s), 1590(w), 1518(s), 1496(w), 1452(m), 1426(m), 1398(s), 1338(s), 1303(s), 1250(s), 1172(s), 1073(s), $1033(\mathrm{~s}) ;[\alpha]^{23}=+15\left(c=0.97, \mathrm{CH}_{2} \mathrm{Cl}_{2}\right)$; MS (ESI) calculated for $\mathrm{C}_{24} \mathrm{H}_{28} \mathrm{O}_{4}[\mathrm{M}+\mathrm{H}]^{+}: 380.2$, found 380.2 .

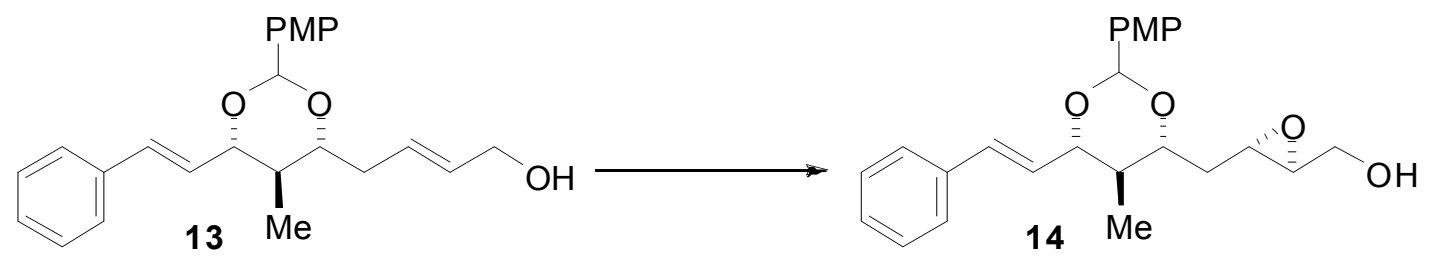


A solution of $\mathrm{Ti}(\mathrm{O}-i-\mathrm{Pr})_{4}(0.107 \mathrm{~mL}, 0.36 \mathrm{mmol})$ in $\mathrm{CH}_{2} \mathrm{Cl}_{2}(1 \mathrm{~mL})$ was cooled to $-78{ }^{\circ} \mathrm{C}$. To this solution was added (+)-diethyl tartrate $(0.074 \mathrm{~mL}, 0.44 \mathrm{mmol})$ and allylic alcohol 13 (138 $\mathrm{mg}, 0.36 \mathrm{mmol})$. A solution of $t$-BuOOH in $\mathrm{CH}_{2} \mathrm{Cl}_{2}(0.15 \mathrm{~mL}, 5.4 \mathrm{M})$ was cooled to $-20{ }^{\circ} \mathrm{C}$ and added via syringe to the reaction. After $8 \mathrm{~h}$ additional $t$ - $\mathrm{BuOOH}$ was added $(0.30 \mathrm{~mL}, 5.4 \mathrm{M})$. After 6 hours the reaction was quenched with a solution of $\mathrm{FeSO}_{4} \cdot 7 \mathrm{H}_{2} \mathrm{O}(736 \mathrm{mg})$ and tartaric acid $(474 \mathrm{mg})$ in water $(2.6 \mathrm{~mL})$. Ether $(3 \mathrm{~mL})$ was added and the reaction mixture was stirred ( $\sim 15$ min) until no peroxides were detected with starch iodide paper. The heterogeneous solution was extracted with ether $(3 \mathrm{x})$ and the combined organics was dried $\left(\mathrm{Na}_{2} \mathrm{SO}_{4}\right)$. The solvent was evaporated and the crude mixture was dissolved in ether $(6.5 \mathrm{~mL})$. This solution was cooled to 0 ${ }^{\circ} \mathrm{C}$ and a solution of $\mathrm{NaOH}$ in brine $(2 \mathrm{~mL}, 30 \% \mathrm{w} / \mathrm{v})$ was added. This solution was stirred for 1 $\mathrm{h}$, extracted with ether $(2 \mathrm{x})$. The combined organic extracts was dried $\left(\mathrm{Na}_{2} \mathrm{SO}_{4}\right)$, evaporated, and purified via column chromatography $(40 \%$ EtOAc/hexanes) to yield $98 \mathrm{mg}$ of white crystalline epoxy alcohol 14 (68\%). ${ }^{1} \mathrm{H}$ NMR $\left(400 \mathrm{MHz}, \mathrm{CDCl}_{3}\right) \delta$ 7.42-7.23 (band, 5H), $7.19\left(\mathrm{AB}, v_{\mathrm{AB}}=\right.$ $227.4, J=8.6,4 \mathrm{H}), 6.69(\mathrm{~d}, J=15.9,1 \mathrm{H}), 6.25(\mathrm{dd}, J=15.9,7.5,1 \mathrm{H}), 5.64(\mathrm{~s}, 3 \mathrm{H}), 4.07(\mathrm{dd}, J=$ 9.3, 8.1, 1H), 3.89 (d, $J=12.4,1 \mathrm{H}), 3.80(\mathrm{~s}, 3 \mathrm{H}), 3.70$ (ddd, $J=9.6,6.2,3.2,1 \mathrm{H}), 3.66-3.59$ (band, 1H), $3.28(\mathrm{~m}, 1 \mathrm{H}), 2.99(\mathrm{~m}, 1 \mathrm{H}), 2.12-1.91$ (band, 3H), 1.87 (ddq, $J=9.8,9.8,6.6,1 \mathrm{H}$ ), $0.89(\mathrm{~d}, J=6.7,3 \mathrm{H}) ;{ }^{13} \mathrm{C} \mathrm{NMR}\left(100 \mathrm{MHz}, \mathrm{CDCl}_{3}\right) \delta 159.883,136.38,133.50,130.96,128.46$, $127.79,127.44,127.35,126.54,113.56,100.32,83.68,79.83,61.82,57.66,55.22,52.78,37.87$, 34.59; IR (thin film) v 3445(br), 2966(m), 2932(m), 2839(m), 2032(w), 1958(w), 1889(w), 1802(w), 1614(s), 1589(w), 1518(s), 1495(w), 1453(m), 1400(s), 1338(s), 1303(s), 1250(s), 1173(s), 1137(s), 1075(s), 1033(s); $[\alpha]^{23}{ }_{\mathrm{D}}=+0.87\left(c=1.2, \mathrm{CH}_{2} \mathrm{Cl}_{2}\right)$; MS (ESI) calculated for $\mathrm{C}_{24} \mathrm{H}_{28} \mathrm{O}_{5}[\mathrm{M}+\mathrm{H}]^{+}:$396.19, found 396.2.

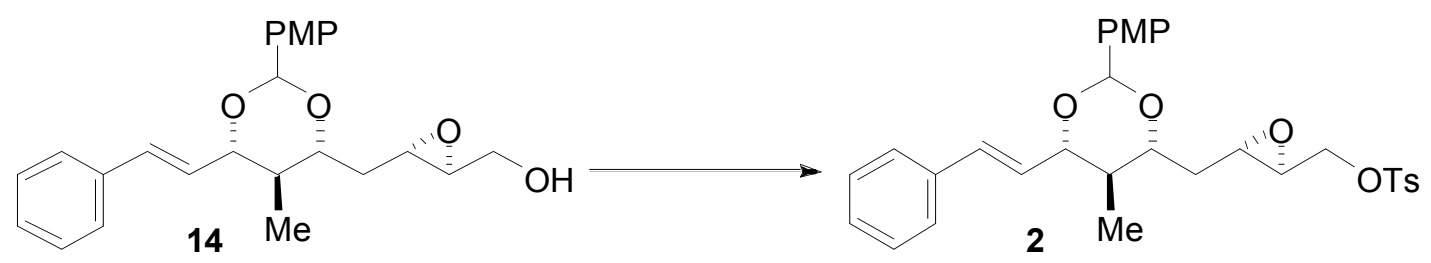

A solution of epoxyl alcohol $14(72 \mathrm{mg}, 0.18 \mathrm{mmol})$ in $\mathrm{CH}_{2} \mathrm{Cl}_{2}(5 \mathrm{~mL})$ was cooled to 0 ${ }^{\circ} \mathrm{C}$. Triethylamine $(0.08 \mathrm{~mL}, 0.6 \mathrm{mmol}), N, N$-dimethylaminopyridine (spatula tip), and toluenesulfonyl chloride $(59 \mathrm{mg}, 0.31 \mathrm{mmol}$ ) were added and the reaction mixture was stirred for $18 \mathrm{~h}$ at $0{ }^{\circ} \mathrm{C}$. Saturated $\mathrm{NH}_{4} \mathrm{Cl}(10 \mathrm{~mL})$ was added to the mixture and it was extracted with $\mathrm{CH}_{2} \mathrm{Cl}_{2}(3 \mathrm{x})$. The combined organics was evaporated and purified via flash chromatography (20\% EtOAc/hexanes) to yield $80 \mathrm{mg}$ of tosylate $2(81 \%) .{ }^{1} \mathrm{H}$ NMR $\left(400 \mathrm{MHz}, \mathrm{CDCl}_{3}\right) \delta 7.55$ $\left(\mathrm{AB}, v_{\mathrm{AB}}=189.0, J=8.2,4 \mathrm{H}\right), 7.40-7.23$ (band, $\left.5 \mathrm{H}\right), 7.17\left(\mathrm{AB}, v_{\mathrm{AB}}=220.9, J=8.6,4 \mathrm{H}\right), 6.67$ $(\mathrm{d}, J=15.9,1 \mathrm{H}), 6.22(\mathrm{dd}, J=15.9,7.6,1 \mathrm{H}), 5.62(\mathrm{~s}, 3 \mathrm{H}), 4.21(\mathrm{dd}, J=11.3,3.7,1 \mathrm{H}), 4.05(\mathrm{dd}$, $J=9.5,7.8,1 \mathrm{H}), 3.95(\mathrm{dd}, J=11.3,6.1,1 \mathrm{H}), 3.80(\mathrm{~s}, 3 \mathrm{H}), 3.75(\mathrm{dd}, J=6.4,6.2,2 \mathrm{H}), 3.69$, (ddd, $J=9.8,6.4,3.2,1 \mathrm{H}), 3.12(\mathrm{~m}, 1 \mathrm{H}), 3.06(\mathrm{~m}, 1 \mathrm{H}), 2.43(\mathrm{~s}, 3 \mathrm{H}), 2.02-1.75$ (band, $5 \mathrm{H}), 0.85(\mathrm{~d}, J=$ 6.6, 3H); ${ }^{13} \mathrm{C}$ NMR $\left(100 \mathrm{MHz}, \mathrm{CDCl}_{3}\right) \delta 159.80,144.88,136.23,133.48,132.43,130.77$, $129.75,128.37,127.80,127.72,127.35,127.13,126.87,126.44,113.46,100.22,83.54,79.49$, $70.09,67.80,55.13,53.81,53.45,37.77,34.24,25.43,21.47,12.15$; IR (thin film) $v 2966(\mathrm{w})$, 2931(w), 2836(w), 1614(w), 1598(w), 1518(m), 1495(w), 1452(w), 1400(w), 1363(s), 1342(m), 1304(w), 1250(s), 1190(s), 1176(s), 1136(w), 1096(m), 1076(m), 1032(m); $[\alpha]^{23}{ }_{\mathrm{D}}=-6.6(c=2.0$, $\mathrm{CH}_{2} \mathrm{Cl}_{2}$ ); MS (ESI) calculated for $\mathrm{C}_{31} \mathrm{H}_{34} \mathrm{O}_{7} \mathrm{~S}[\mathrm{M}+\mathrm{H}]^{+}:$550.2, found 550.2. 


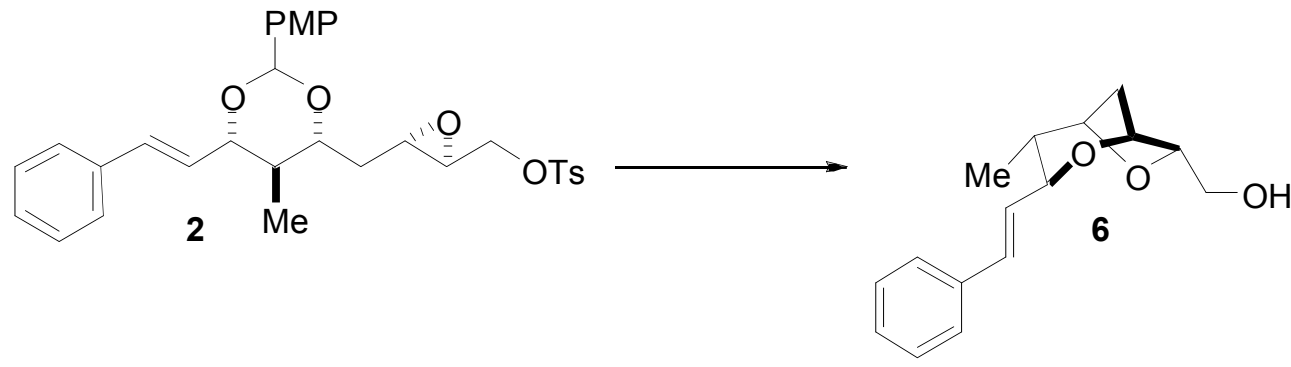

To a solution of tosylate $2(40 \mathrm{mg}, 0.073 \mathrm{mmol})$ in THF $(2 \mathrm{~mL})$ was added $10 \% \mathrm{HCl}(1$ $\mathrm{mL})$. Methanol $(\sim 3 \mathrm{~mL})$ was added to the cloudy mixture until it became clear. The reaction mixture was stirred at $\mathrm{rt}$ for $18 \mathrm{~h}$. To the reaction mixture was added $10 \% \mathrm{NaOH}(\sim 1 \mathrm{~mL})$ until it became basic. After $5 \mathrm{~min} 10 \% \mathrm{HCl}(\sim 1 \mathrm{~mL})$ was added until the solution became acidic and the reaction mixture was stirred for $4 \mathrm{~h}$. The reaction was extracted with $\mathrm{CH}_{2} \mathrm{Cl}_{2}(3 \mathrm{x})$, dried $\left(\mathrm{MgSO}_{4}\right)$, and purified via flash chromatography $(50 \% \mathrm{EtOAc} / \mathrm{hexanes})$ to yield $12 \mathrm{mg}$ of bicyclic ether $6(62 \%)$. ${ }^{1} \mathrm{H}$ NMR $\left(400 \mathrm{MHz} \mathrm{CDCl}_{3}\right) \delta 7.40-7.20(\mathrm{~m}, 5 \mathrm{H}), 6.58(\mathrm{~d}, J=15.8,1 \mathrm{H})$, $6.12(\mathrm{dd}, J=15.8,7.5,1 \mathrm{H}), 4.45(\mathrm{~s}, 1 \mathrm{H}), 4.32(\mathrm{~d}, J=6.6,1 \mathrm{H}), 4.12-4.01$ (band, 3H), 3.96 (m, $1 \mathrm{H}), 2.08$ (ddd, $J=11.7,6.6,2.6,1 \mathrm{H}), 1.96-1.93$ (band, $2 \mathrm{H}), 1.54$ (dq, $J=6.8,6.8,1 \mathrm{H}), 0.94$ (d, $J=6.7,3 \mathrm{H}) ;{ }^{13} \mathrm{C}$ NMR $\left(100 \mathrm{MHz}, \mathrm{CDCl}_{3}\right) \delta 136.30,132.34,128.86,128.34,127.58,126.36$, $82.71,79.61,78.79,74.26,60.80,41.50,38.63,15.09$; IR (thin film) v 3415(br), 2962(s), 2931(s), 2875(s), 2245(w), 1494(m), 1450(s), 1374(m), 1343(m), 1220(m), 1144(s), 1106(m), 1031(s), 1007(s); $[\alpha]^{22}{ }_{\mathrm{D}}=-42\left(c=0.58, \mathrm{CH}_{2} \mathrm{Cl}_{2}\right)$; MS (ESI) calculated for $\mathrm{C}_{16} \mathrm{H}_{20} \mathrm{O}_{3}[\mathrm{M}+\mathrm{H}]^{+}$: 260.33 , found 260.3

${ }^{1}$ Evans, D. A.; Downey, C. W.; Shaw, J. T.; Tedrow, J. S. Org. Lett. 2002, 4, 1127. 